\title{
Development of Magnetic Particle Distribution Imaging Using Magnetic Field Reconstruction for Biopsy of the Sentinel Lymph Node
}

\author{
Akari Inagaki ${ }^{1}$, Tomoko Suzuki ${ }^{1}$, Yuki Mima ${ }^{2}$ and Kenjiro Kimura ${ }^{2,3,4, *}$ \\ 1 Graduate School of Science, Kobe University, Hyogo 657-8501, Japan; inagaki_akari@stu.kobe-u.ac.jp (A.I.); \\ bravo_violoncello@yahoo.co.jp (T.S.) \\ 2 Integral Geometry Science Co., Ltd., Hyogo 657-8501, Japan; ymima@ig-instrum.co.jp \\ 3 Center for Mathematical and Data Sciences, Kobe University, Hyogo 657-8501, Japan \\ 4 Japan Agency for Medical Research and Development, Tokyo 100-0004, Japan \\ * Correspondence: kimura@gold.kobe-u.ac.jp
}

\section{check for} updates

Citation: Inagaki, A.; Suzuki, T.; Mima, Y.; Kimura, K. Development of Magnetic Particle Distribution Imaging Using Magnetic Field Reconstruction for Biopsy of the Sentinel Lymph Node. Magnetochemistry 2021, 7, 85. https://doi.org/10.3390/ magnetochemistry7060085

Academic Editors: Paolo Silvestrini and Carmine Granata

Received: 19 May 2021

Accepted: 9 June 2021

Published: 15 June 2021

Publisher's Note: MDPI stays neutral with regard to jurisdictional claims in published maps and institutional affiliations.

Copyright: (c) 2021 by the authors. Licensee MDPI, Basel, Switzerland. This article is an open access article distributed under the terms and conditions of the Creative Commons Attribution (CC BY) license (https:/ / creativecommons.org/licenses/by/ $4.0 /)$.

\begin{abstract}
The sentinel lymph node is the first lymph-node-draining cancer metastasis. The identification of the sentinel lymph node using magnetic particles and a magnetic sensor has attracted attention in recent years, as this method is less invasive than the conventional method of radiotracer injection. However, the development of a two-dimensional measurement method for sentinel lymph nodes using magnetic nanoparticles remains an issue. In the present study, a method and apparatus for the two-dimensional imaging of magnetic particle distribution were developed to detect a lymph node with magnetic particles concentrated within lymphoid tissues. The method comprises the reconstruction of the magnetic field measured with a high-sensitivity magnetic sensor and with a magnetic detection ability of $2 \mathrm{nT} / \sqrt{ } \mathrm{Hz}$ at $100 \mathrm{~Hz}(5 \mathrm{nT} / \sqrt{ } \mathrm{Hz}$ at $1 \mathrm{~Hz})$. The proposed system measures the two-dimensional magnetic field distribution in an area of up to $25 \times 25 \mathrm{~mm}^{2}$ using a coil generating a $0.77 \mathrm{mT}$ external magnetic field applied to the measurement target. The improved spatial resolution of the images makes it possible to use two-dimensional imaging for diagnostics of breast cancer metastases.
\end{abstract}

Keywords: cancer metastases; sentinel lymph node; imaging; magnetic nanoparticles; spatial resolution

\section{Introduction}

Breast cancer is a disease that includes the growth of cancerous cells and the formation of new blood and lymphatic vessels within these cells from the existing surrounding blood vessels and lymphatic vessels. Because new blood vessels and lymphatic vessels are formed within the tumor, free-floating cancer cells from the breast cancer are transported throughout the body by these vessels and develop metastases at new locations. Specifically, lymph nodes that filter impurities in lymphatic vessels are the most susceptible tissues to cancer cell accumulation and the formation of metastases. A lymph node where the breast cancer first metastasizes is called the sentinel lymph node (SLN). Before breast cancer surgery, sentinel lymph node biopsy is performed in order to confirm whether the cancer has metastasized or not [1-5]. There are multiple lymph nodes in lymphoid tissues [1], and the average diameter of a lymph node is approximately $1 \mathrm{~cm}$ [4]. At present, the SLN is usually identified by dye-guided [2,3] and radioisotope (RI) methods [6], but the latter method is highly invasive, as a radioactive material is injected into lymphoid tissues as a tracer [7]. In recent years, the identification of the SLN using magnetic particle sensors has attracted attention due to its low invasiveness compared to the conventional methods using radiotracers $[8,9]$. Nanoparticles $[10,11]$ of ferromagnets materials, such as magnetite $\left(\mathrm{Fe}_{3} \mathrm{O}_{4}\right)$ and ferrite $\left(\mathrm{MO} \cdot \mathrm{Fe}_{2} \mathrm{O}_{3}, \mathrm{MO}\right.$ : metal oxide) possessing superparamagnetic properties that exhibit magnetism and produce heat in response to electromagnetic waves, are already 
being used in the field of medicine [12]. With the existing dye-guided RI or magnetic particle methods, positions of metastases are manually confirmed using a Geiger counter and magnetic probe; thus, the results strongly depend on the subjective judgment of technicians, and it is difficult to directly ascertain the area of tracer distribution. In addition, identifying the SLN with the highest tracer content in all lymph nodes is challenging to experts (Figure 1a,b). This issue can be resolved by measuring the two-dimensional distribution of tracers in all lymph nodes (Figure 1c,d).
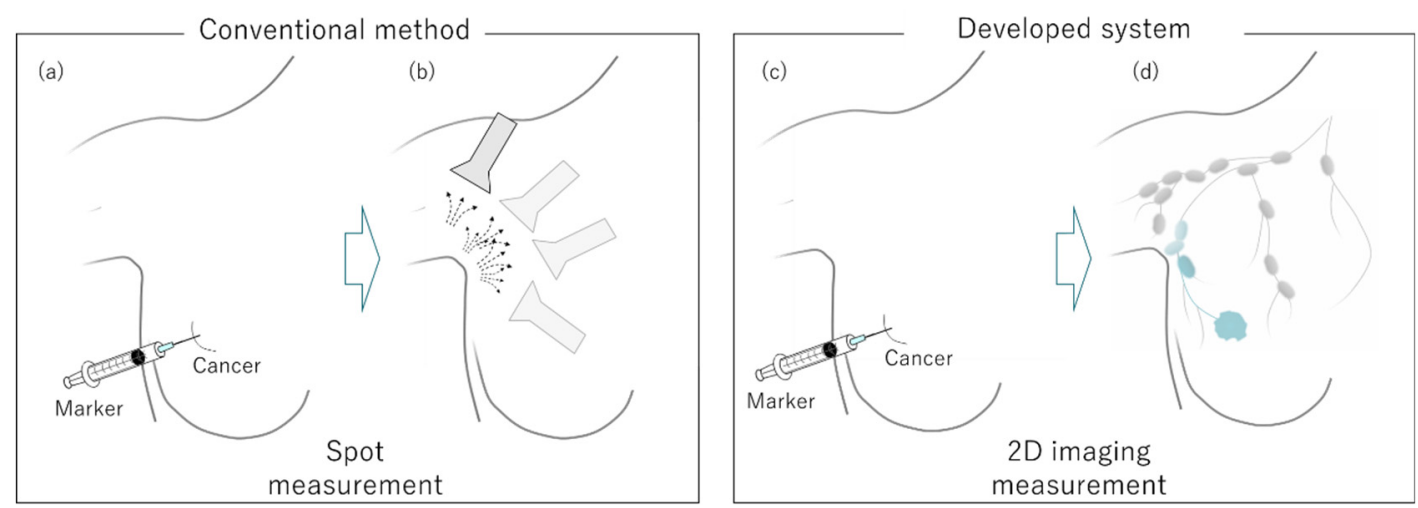

Figure 1. Methods of the sentinel lymph node identification. The position of a tracer injected into a breast cancer (a) in the conventional method is manually detected with a detector by the location of the sentinel lymph node (b). According to the proposed method, magnetic particles are injected as a tracer to the breast cancer (c), and then the spatial distribution of the magnetic particles is determined by a two-dimensional scan with a magnetic sensor (d).

Thus, in the present work, we developed a system and apparatus to measure the two-dimensional distribution of magnetic particles using image reconstruction and a high-sensitivity magnetic sensor and demonstrated the system's performance.

\section{Results}

Figure 2 demonstrates the result of a verification experiment of image reconstruction using a ferrite magnet. Figure $2 \mathrm{a}, \mathrm{b}$ demonstrates a magnetic field distribution measured at 1.5 and $1.7 \mathrm{~mm}$ from the ferrite magnet surface. Figure $2 \mathrm{c}$ is a reconstructed image using the two magnetic field distributions in Figure 2a,b. The magnetic field distribution images shown in Figure 2a,b detected three ferrite magnets as one object, as they could not differentiate them. However, in the reconstructed image in Figure 2c, the spatial resolution is improved, and the three ferrite magnets can be easily distinguished.

(a) $1.7 \mathrm{~mm}$

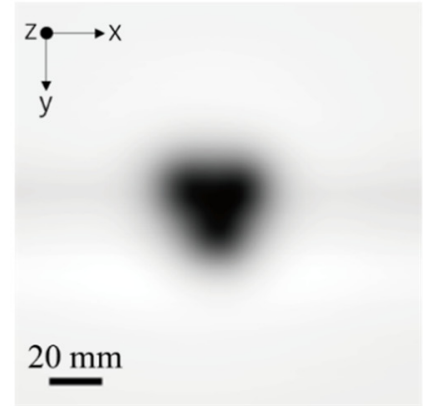

(b) $1.5 \mathrm{~mm}$

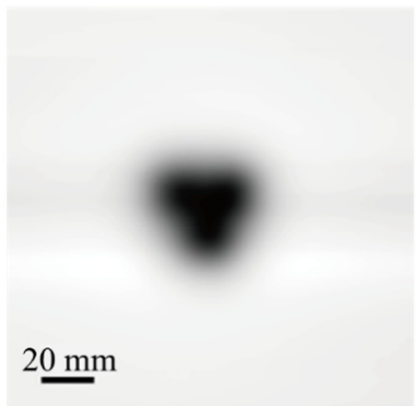

(c) Reconstructed image

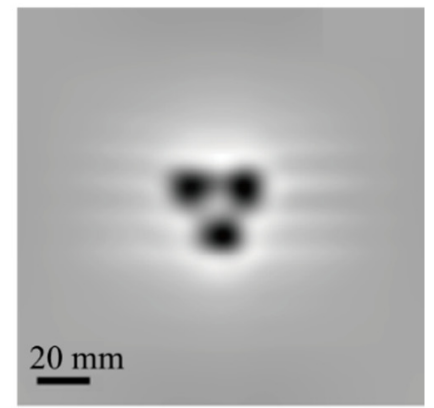

Figure 2. Verification experiments of image reconstruction: magnetic field distribution measured at $1.7 \mathrm{~mm}(\mathbf{a})$ and $1.5 \mathrm{~mm}(\mathbf{b})$ above the ferrite magnet sample surface; reconstruction (c) based on images $(\mathbf{a}, \mathbf{b})$.

Figure 3 shows the experimental results of experiments I to III, where magnetic particles were measured. The measured magnetic field distributions are demonstrated in 
Figure $3 a, b$, respectively, while Figure $3 c$ shows the reconstructed image. In the present experiment, when the distance from the sample surface to the magnetic sensor was $2.0 \mathrm{~mm}$ or more, the sample shape could not be visible in the obtained images without reconstruction. In addition, in the experiments where the distance from the magnetic particle sample surface to the magnetic sensor was $4.0 \mathrm{~mm}$ or more, a cross-shaped sample could not be distinguished on the image without reconstruction; hence, a magnetic particle distribution could not be detected. In contrast, in the reconstructed images, even in Figure 3II,III, where the shape of the sample cannot be distinguished in the experimental images, the spatial resolution of the reconstructed image is improved to the rate that the sample shape can be distinguished.

(a) $2.0 \mathrm{~mm}$

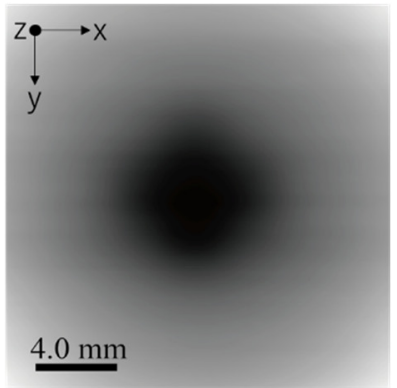

II
(a) $3.0 \mathrm{~mm}$

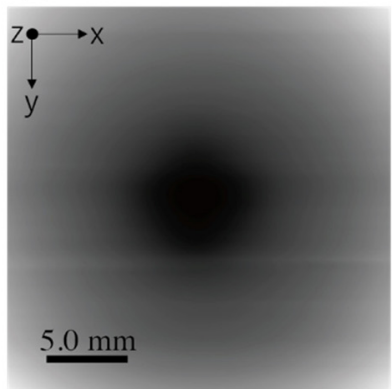

III

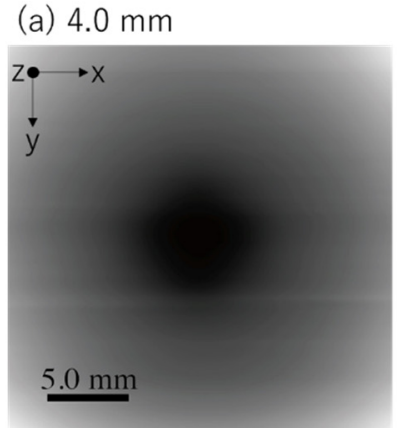

(b) $1.0 \mathrm{~mm}$

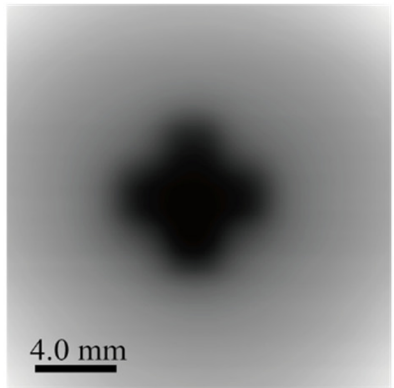

(b) $2.0 \mathrm{~mm}$

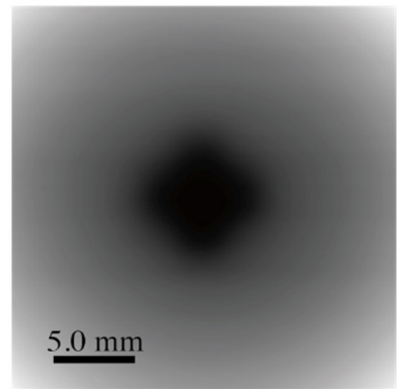

(b) $3.0 \mathrm{~mm}$

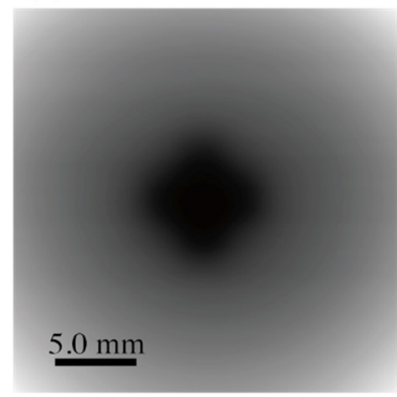

(c) Reconstructed image

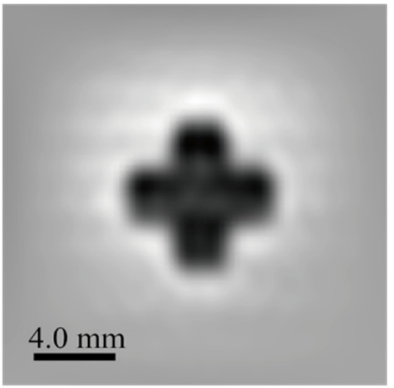

(c) Reconstructed image

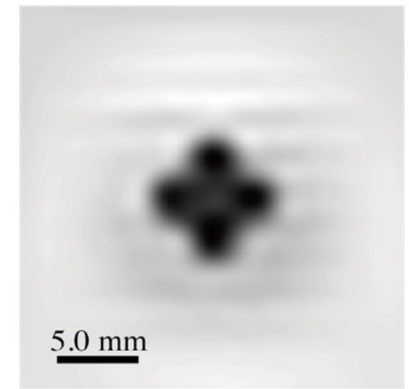

(c) Reconstructed image

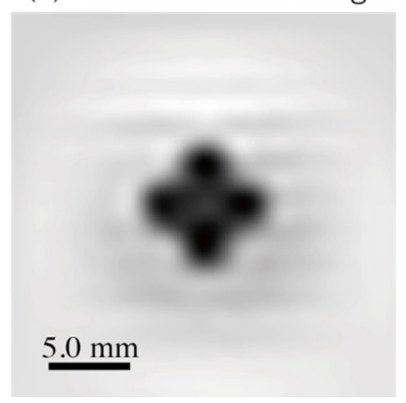

Figure 3. Two-dimensional magnetic particle distributions (I, II, and III): (a,b) measurement image; (c) reconstruction image based on $(\mathbf{a}, \mathbf{b})$.

\section{Discussion}

Figure 4 shows magnetic field profiles along the yellow dashed line in the image reconstruction verification experiment using ferrite magnets as the target. In the profiles, the differences between the magnetic field intensities inside and outside of the ferrite magnet's location (Width 1) and the difference between the intensities on ferrite magnets and between them are shown (Width 2). Relative ratios of Width 2 compared to Width 1 in Figure $4 \mathrm{a}, \mathrm{b}$ are $0.5 \%$ and $4.3 \%$, but in Figure $4 \mathrm{c}$, it is increased up to $51 \%$. Therefore, the spatial resolution was improved using the image reconstruction to a rate providing easy identification of the magnetic source shape. 
(a) $1.7 \mathrm{~mm}$

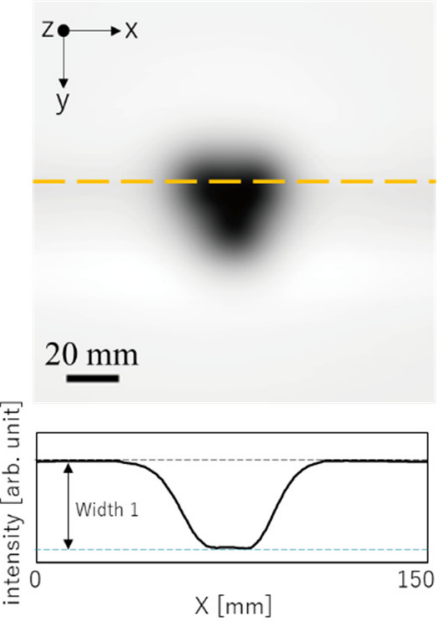

(b) $1.5 \mathrm{~mm}$

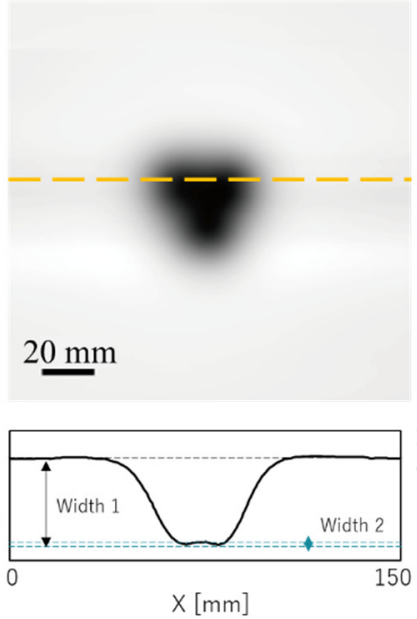

(c) Reconstructed image

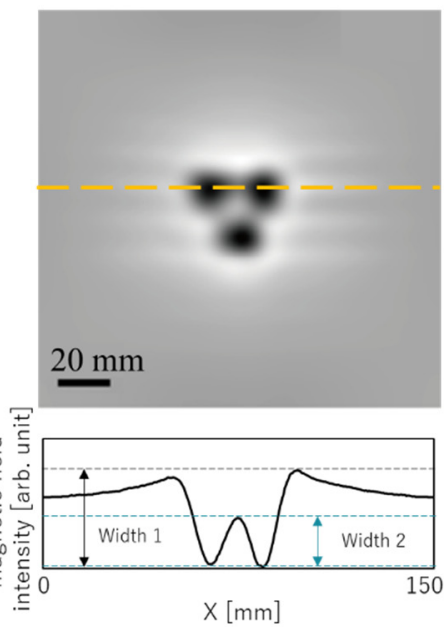

Figure 4. Two-dimensional magnetic particle distributions: I images and magnetic field intensity profiles measured at $1.7 \mathrm{~mm}(\mathbf{a})$ and $1.5 \mathrm{~mm}(\mathbf{b})$ distances from the magnetic source, and reconstruction I (c) based on Images I (a) and I (b).

Figure 5 shows the image and line profiles of the sample with embedded magnetic particles. The half-width on the line profile is 9.5, 7.2, and $3.0 \mathrm{~mm}$ in Figure $5 \mathrm{a}-\mathrm{c}$, respectively. As the sample width along the line was $2.5 \mathrm{~mm}$, the spatial resolution was improved using the electromagnetic field reconstruction. Figure $6 \mathrm{c}$ demonstrates a superposition of reconstructed and real images of the measured target. The shape of the magnetic particle distribution delineated in the reconstruction was equivalent to the actual sample shape. Therefore, the two-dimensional magnetic particle distribution measurement system utilizing image reconstruction and a high-sensitivity magnetic sensor enhances the image spatial resolution and provides clear imaging of magnetic particle distribution.

(a) $2.0 \mathrm{~mm}$

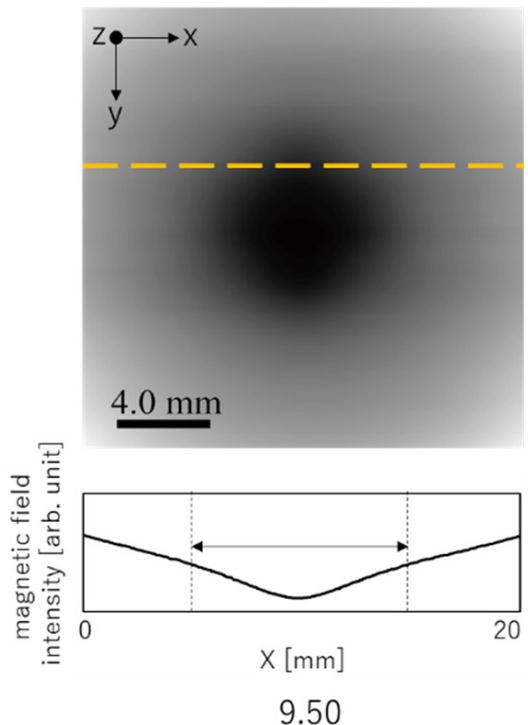

(b) $1.0 \mathrm{~mm}$

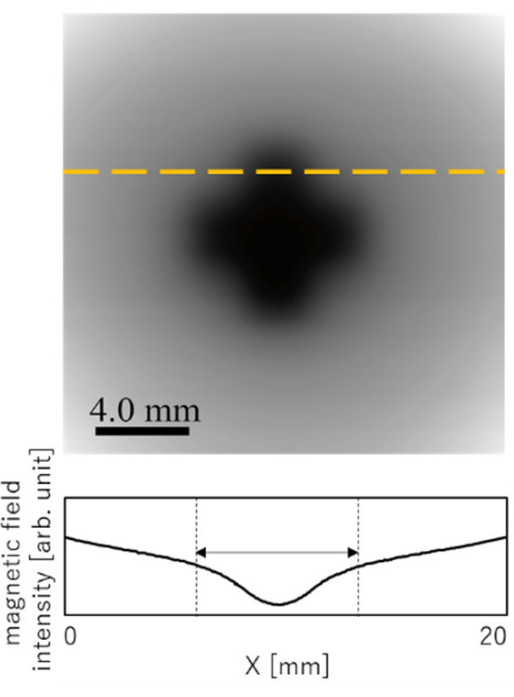

7.21 (c) Reconstructed image

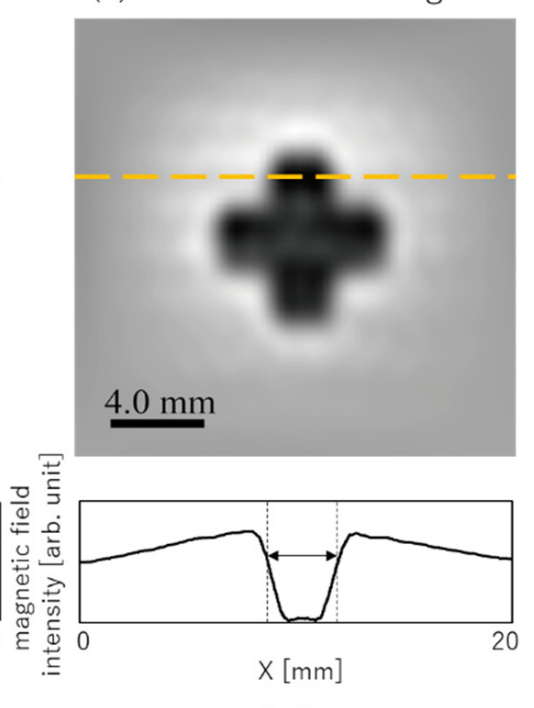

3.06

Figure 5. Two-dimensional magnetic particle distributions: I images and magnetic field intensity profiles measured at $2.0 \mathrm{~mm}(\mathbf{a})$ and $1.0 \mathrm{~mm}(\mathbf{b})$ from the magnetic source, and reconstruction I (c) based on Images I (a) and I (b). 
(a)

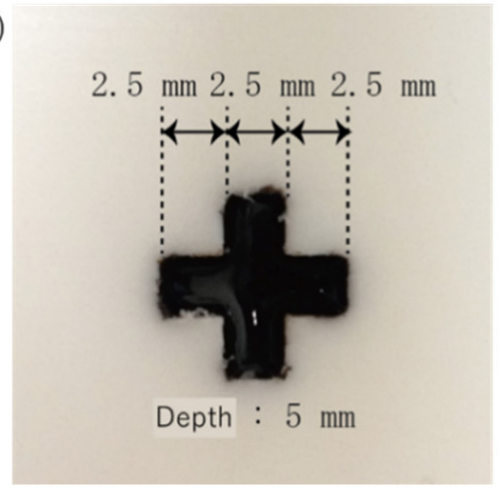

(b)

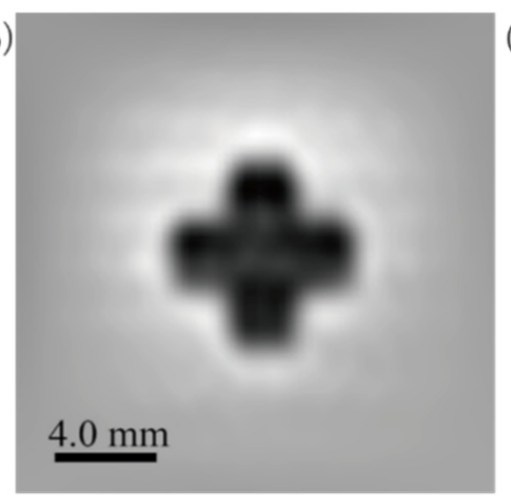

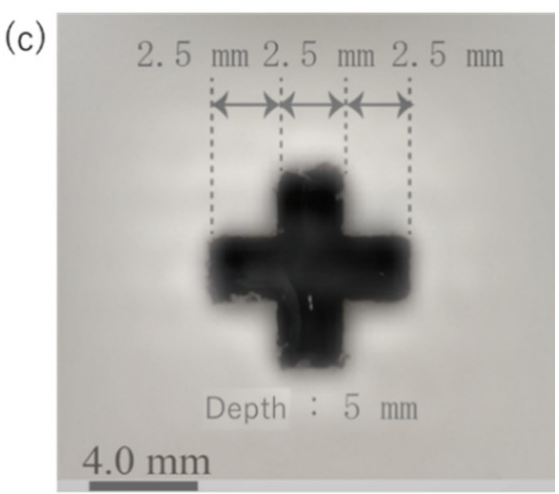

Figure 6. Comparison of the sample photo and its image reconstruction; (c) overlapped (a,b) images.

\section{Materials and Methods}

The scheme of a two-dimensional magnetic particle distribution measurement system using image reconstruction and a high-sensitivity magnetic sensor is shown in Figure 7. The developed system consists of hardware that collects the measurement data and image reconstruction software for their analysis. The hardware consists of a high-sensitivity magnetic sensor mounted on an $X-Y-Z$ triaxial positioning stage with stepping motors, a coil generating an external magnetic field, a power source for the coil, a signal amplifier, an $\mathrm{AD}$ converter, and a computer to process the signals. As the magnetic sensor, we used the STJ-220 tunneling magnetoresistance device from MicroMagnetics (TMR has a magnetic detection ability of $2 \mathrm{nT} / \sqrt{ } \mathrm{Hz}$ at $100 \mathrm{~Hz}(5 \mathrm{nT} / \sqrt{ } \mathrm{Hz}$ at $1 \mathrm{~Hz}))$. The external field was generated by a Helmholtz coil with a diameter of $8 \mathrm{~cm}$ consisting of $2 \times 38$ turns of a $0.35 \mathrm{~mm}$ enamel wire. The 1.0 A direct current was applied to the Helmholtz coil to create an external electric field. The measured sample was placed at the center of the coil. When the measured surface is distant from the magnetic source, the measured magnetic field distribution has a poor spatial resolution. However, this problem was solved using image reconstruction. The magnetic field distribution within the sample was reconstructed from the measurement results by an analytical solution of the governing equation of the static magnetic field (Equation (1)). A variety of magnetic field reconstruction methods have been developed [13-15]. Roth's method [13] takes the magnetic sources at only one side of the two half-spaces divided by the measurement plane. Our method takes the magnetic sources at both sides of the two half-spaces divided by the measurement plane. This provided us with images of magnetic particle distribution within the samples with a high spatial resolution.

The image reconstruction procedure used in the present study is introduced below [16]. As the space between the magnetic sensor and magnetic particles does not contain other magnetic sources, the governing equation of the magnetic field in the free space is expressed by Equation (1):

$$
\Delta H_{i}(x, y, z)=0(i=x, y, z)
$$

Among magnetic field vectors in a Cartesian coordinate system $(x, y, z)$, in the present study, we measure the magnetic field distribution in the $x-y$ plane along the $z$-direction (see Figure 7). The solution for the $H_{z}$ component in Equation (1) is as follows;

$$
H_{z}(x, y, z)=\frac{1}{(2 \pi)^{2}} \iint_{-\infty}^{\infty} e^{i k_{x} x+i k_{y} y}\left\{a\left(k_{x}, k_{y}\right) e^{z \sqrt{k_{x}^{2}+k_{y}{ }^{2}}}+b\left(k_{x}, k_{y}\right) e^{-z \sqrt{k_{x}{ }^{2}+k_{y}{ }^{2}}}\right\} d k_{x} d k_{y}
$$

where $k_{x}$ and $k_{y}$ are wavenumbers in the $x$ - and $y$-directions, respectively. By determining $a\left(k_{x}, k_{y}\right)$ and $b\left(k_{x}, k_{y}\right)$ from Equation (2), $H_{z}(x, y, z)$ can be reconstructed. $a\left(k_{x}, k_{y}\right)$ and $b\left(k_{x}, k_{y}\right)$ are calculated from the two-dimensional magnetic field distribution at $z=0$, $H_{z}(x, y, 0)$, and two-dimensional magnetic field gradient distribution, $\partial H_{z}(x, y, z) /\left.\partial z\right|_{z=0}$ are set as the boundary conditions. Equation (3) is derived by substitution of $a\left(k_{x}, k_{y}\right)$ and 


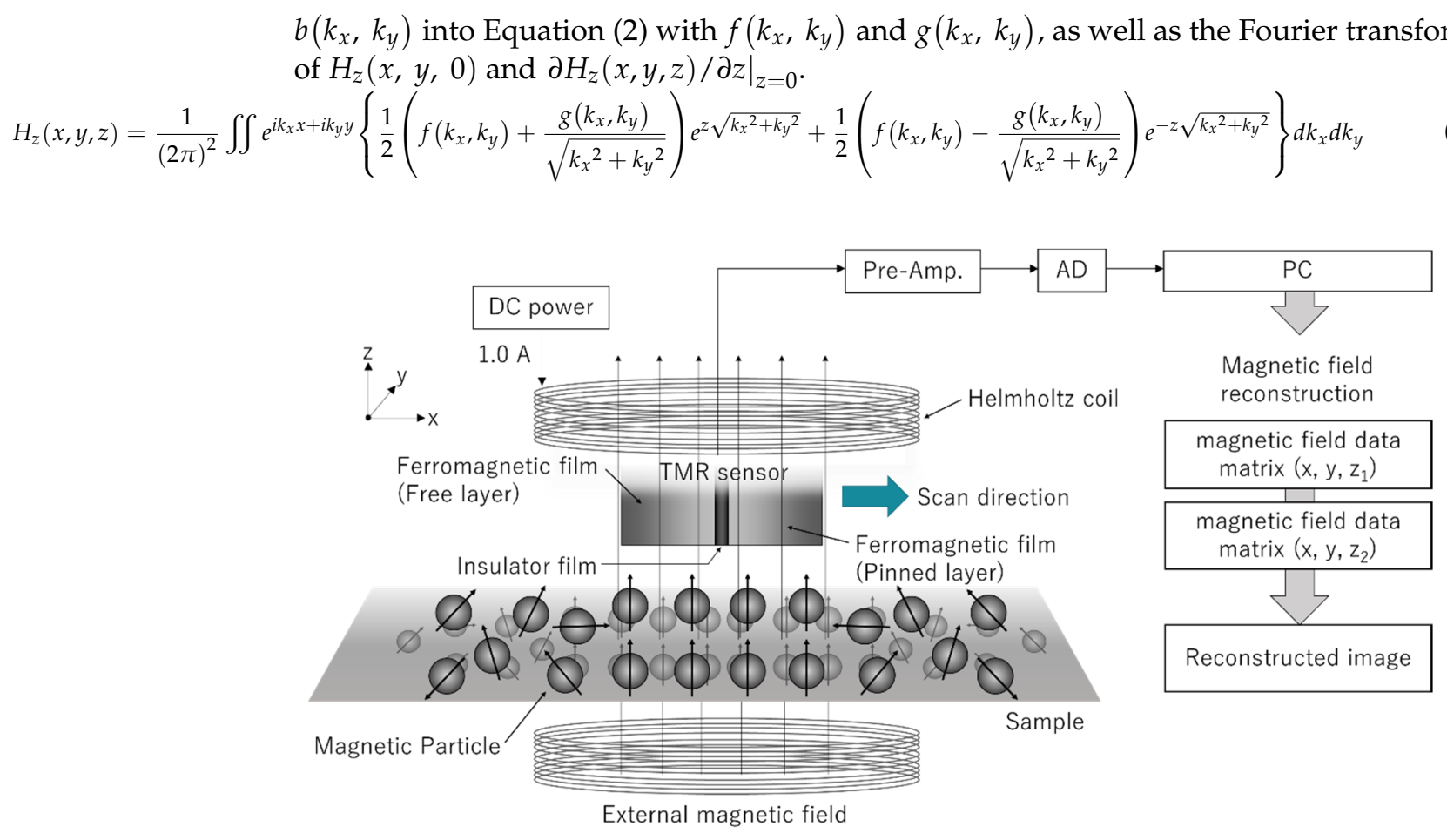

Figure 7. Two-dimensional magnetic particle distribution measurement system utilizing image reconstruction and a high-sensitivity magnetic sensor. A magnetic field from the Helmholtz coil is applied to magnetic particles, while a magnetic sensor scans magnetic field distribution along the surface using the stepping motor. The Helmholtz coil is not used in the case of a magnet not requiring magnetization. Image reconstruction was performed on the basis of obtained magnetic field measurement data.

Therefore, magnetic field distribution in the free space can be reconstructed using the measured distributions of the two-dimensional magnetic field and its gradient. In the present system, these distributions were determined by measuring two two-dimensional magnetic field distributions at different $z$ positions.

In this paper, we firstly performed the image reconstruction with a ferrite magnet used as the measurement target. This experiment was conducted for the purpose of verifying the performance of the developed system. The ferrite magnet exhibits residual magnetization, so the experiment was conducted with the Helmholtz coil removed from the measurement system shown in Figure 7. First, in order to confirm the performance of the developed system, we measured a general magnet without applying a magnetic field. For the measurements, we used a sample comprising circular ferrite magnets with a diameter of $6 \mathrm{~mm}$ placed at the top of an equilateral triangle with a side length of $2 \mathrm{~cm}$ (Figure 8). Measurements were taken at distances of 1.5 and $1.7 \mathrm{~mm}$ from the TMR sensor to the sample surface. The images had a $128 \times 128$ pixel resolution, while the scanning area was $150 \times 150 \mathrm{~mm}^{2}$.

We then registered the response from magnetic particles magnetized by the coil and examined the two-dimensional magnetic particle distribution measurement system utilizing image reconstruction of high-sensitivity magnetic sensor data. Figure 9 shows the structure of the studied sample. Magnetic particles with a diameter of $10 \mathrm{~nm}$ were dispersed within the organic solvent. The saturation magnetization was $44 \mathrm{mT}$, and the initial magnetic susceptibility was 2.64. Measurements were taken at the following six conditions. Density at $25^{\circ} \mathrm{C}$ was $1.21 \times 10^{3} \mathrm{~kg} / \mathrm{m}^{3}$, and magnetic particle concentration was $7.9 \mathrm{vol} \%$. 
(a)

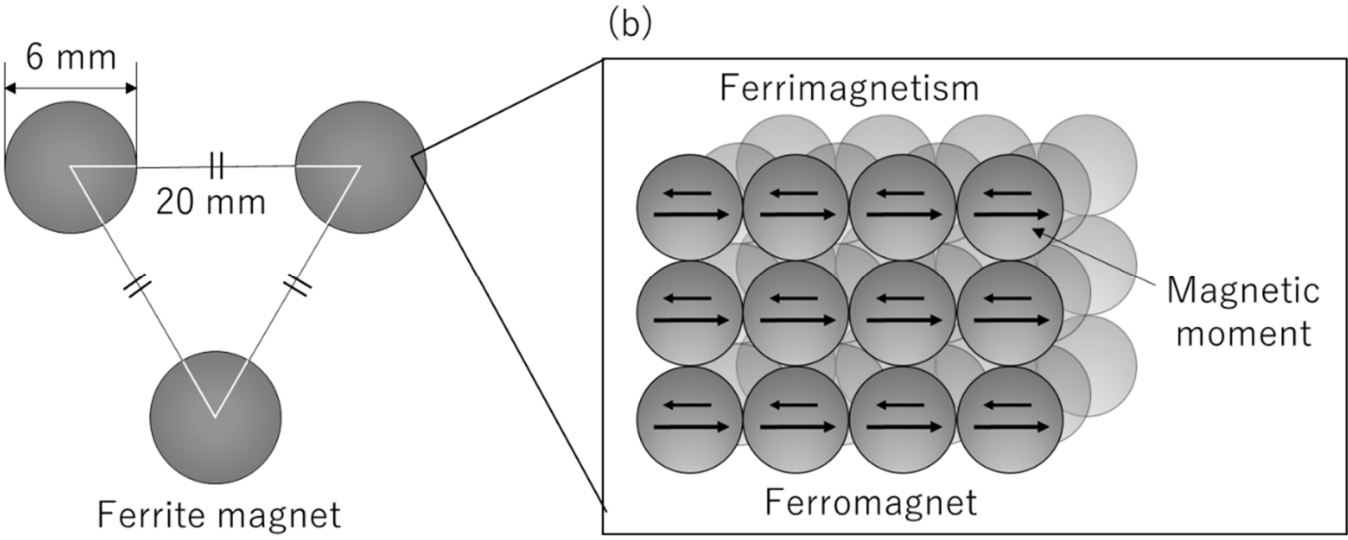

Figure 8. A ferrite magnet sample used in the measurements: schematic of the sample (a) and its structure (b).

(a)

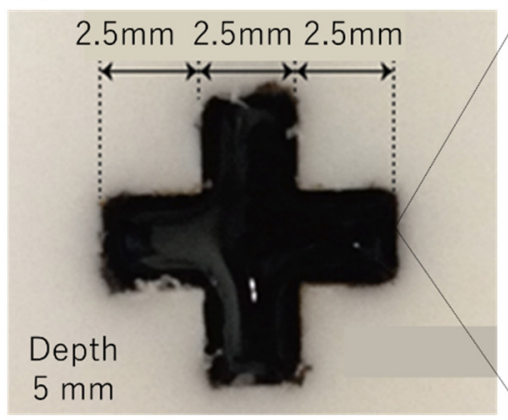

(b)

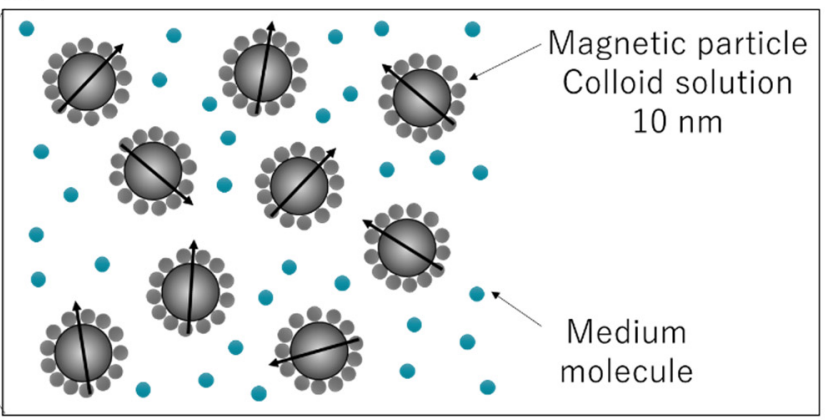

Figure 9. A magnetic-particle-containing sample used in the measurements: (a) optical image of the sample; (b) schematic of the sample structure.

I (a) Distance between sensor and sample: $2.0 \mathrm{~mm}$, scanning area: $20 \mathrm{~mm} \times 20 \mathrm{~mm}$. I (b) Distance between sensor and sample: $1.0 \mathrm{~mm}$, scanning area: $20 \mathrm{~mm} \times 20 \mathrm{~mm}$. II (a) Distance between sensor and sample: $3.0 \mathrm{~mm}$, scanning area: $20 \mathrm{~mm} \times 20 \mathrm{~mm}$. II (b) Distance between sensor and sample: $2.0 \mathrm{~mm}$, scanning area: $20 \mathrm{~mm} \times 20 \mathrm{~mm}$. Ш(a) Distance between sensor and sample: $4.0 \mathrm{~mm}$, scanning area: $25 \mathrm{~mm} \times 25 \mathrm{~mm}$. Ш(b) Distance between sensor and sample: $3.0 \mathrm{~mm}$, scanning area: $25 \mathrm{~mm} \times 25 \mathrm{~mm}$.

Under these six conditions, the image resolution was $64 \times 64$ pixels.

\section{Conclusions}

The two-dimensional magnetic particle distribution measurement system utilizing image reconstruction and a high-sensitivity magnetic sensor provides a two-dimensional visualization of magnetic particle distribution with a high spatial resolution, even when the magnetic source is distant from the measurement surface. Therefore, the SLN can be detected with magnetic particles injected into the body using a magnetic sensor located near the skin surface. In this paper, the maximum distance between the magnetic sensor and the magnetic source was $4 \mathrm{~mm}$. When the distance between the magnetic sensor and the magnetic source is far, low-spatial-frequency components in the Fourier-transformed data of the magnetic field distribution expand to the far side, but the high-spatial-frequency components become weak. Therefore, to achieve high spatial resolution at a distance, it is important to detect the weak high-spatial-frequency components [17].

Following the obtained results, the studies are in progress toward the application of a method for the biopsy of the SLN. 
Author Contributions: A.I., T.S., Y.M., and K.K. were involved in the planning of the experiments, the implementation of the experiments, and the data analysis. A.I., T.S., Y.M., and K.K. were involved in the data interpretation. All authors have read and agreed to the published version of the manuscript.

Funding: Japan Agency for Medical Research and Development.

Institutional Review Board Statement: Not applicable.

Informed Consent Statement: Not applicable.

Data Availability Statement: Not applicable.

Acknowledgments: We thank Crimson Interactive Pvt. Ltd. (Ulatus, Maharashtra, India)—www. ulatus.jp for their assistance in manuscript translation and editing.

Conflicts of Interest: To the best of our knowledge, the named authors have no conflict of interest, financial or otherwise.

\section{References}

1. D'Angelo-Donovan, D.D.; Dickson-Witmer, D.; Petrelli, N.J. Sentinel lymph node biopsy in breast cancer: A history and current clinical recommendations. J. Surg. Oncol. 2012, 21, 196-200. [CrossRef] [PubMed]

2. Ahmed, M.; Purushotham, A.D.; Douek, M. Novel techniques for sentinel lymph node biopsy in breast cancer: A systematic review. Lancet Oncol. 2014, 15, e351-e362. [CrossRef]

3. Giuliano, A.E.; Kirgan, D.M.; Guenther, J.M.; Morton, D.L. Lymphatic mapping and sentinel lymphadenectomy for breast cancer. Ann. Surg. 1994, 220, 391-401. [CrossRef] [PubMed]

4. Lymphoid tissues. In Gray's Anatomy: The Anatomical Basis of Clinical Practice, 41st ed.; Standring, S. (Ed.) Elsevier: Philadelphia, PA, USA, 2016; Volume 4, pp. 73-74.

5. Morton, D.L.; Wen, D.; Wong, J.H.; Economou, J.S.; Cagle, L.A.; Storm, F.K.; Foshag, L.J.; Cochran, A.J. Technical details of intraoperative lymphatic mapping for early stage melanoma. Arch. Surg. 1992, 127, 392-399. [CrossRef] [PubMed]

6. Krag, D.N.; Weaver, D.L.; Alex, J.C.; Fairbank, J.T. Surgical resection and radiolocalization of the sentinel lymph node in breast cancer using a gamma probe. Surg. Oncol. 1993, 2, 335-340. [CrossRef]

7. Linehan, D.C.; Hill, A.D.K.; Akhurst, T.; Yeung, H.; Yeh, S.D.; Tran, K.N.; Borgen, P.I.; Cody, H.S., III. Intradermal radiocolloid and intraparenchymal blue dye injection optimize sentinel node identification in breast cancer patients. Ann. Surg. Oncol. 1999, 6, 450-454. [CrossRef] [PubMed]

8. Shiozawa, M.; Lefor, A.T.; Hozumi, Y.; Kurihara, K.; Sata, N.; Yasuda, Y.; Kusakabe, M. Sentinel lymph node biopsy in patients with breast cancer using superparamagnetic iron oxide and a magnetometer. Breast Cancer 2013, 20, 223-229. [CrossRef] [PubMed]

9. Sekino, M.; Kuwahata, A.; Ookubo, T.; Shiozawa, M.; Ohashi, K.; Kaneko, M.; Saito, I.; Inoue, Y.; Ohsaki, H.; Takei, H.; et al. Handheld magnetic probe with permanent magnet and Hall sensor for identifying sentinel lymph nodes in breast cancer patients. Sci. Rep. 2018, 8, 1195. [CrossRef] [PubMed]

10. Lu, A.-H.; Salabas, E.; Schüth, F. Magnetic nanoparticles: Synthesis, protection, functionalization, and application. Angew. Chem. Int. Ed. 2007, 46, 1222-1244. [CrossRef] [PubMed]

11. Salata, O. Applications of nanoparticles in biology and medicine. J. Nanobiotechnology 2004, 2, 3. [CrossRef] [PubMed]

12. Mornet, S.; Vasseur, S.; Grasset, F.; Veverka, P.; Goglio, G.; Demourgues, A.; Portier, J.; Pollert, E.; Duguet, E. Magnetic nanoparticle design for medical applications. Prog. Solid State Chem. 2006, 32, 237-247. [CrossRef]

13. Roth, B.J.; Sepulveda, N.G.; Wikswo, J.P. Using a magnetometer to image a two-dimensional current distribution. J. Appl. Phys. 1989, 65, 361-372. [CrossRef]

14. Shi, R.; Jung, J.-H.; Schweser, F. Twodimensional local Fourier image reconstruction via domain decomposition Fourier continuation method. PLoS ONE 2019, 14, e0197963. [CrossRef] [PubMed]

15. Liyakathunisa; Ravi Kumar, C.N. A novel super resolution reconstruction of low reoslution images progressively using dct and zonal filter based denoising. Int. J. Comput. Sci. Inf. Technol. 2011, 3, 1. [CrossRef]

16. Mima, Y.; Kimura, K.; Inao, T.; Oyabu, N.; Kimura, N. Failure analysis of electric circuit board by high resolution magnetic field microscopy. In Proceedings of the 2013 3rd IEEE CPMT Symposium Japan, Kyoto, Japan, 11-13 November 2013; pp. 1-4.

17. Kimura, K.; Mima, Y.; Inao, T.; Oyabu, N.; Inao, T.; Kimura, N. Development of High-resolution Magnetic Imaging Method with Tunneling Magnetoresistance Device to Inspect Reinforcing Steels Inside a Concrete Building. J. Jpn. Soc. Non-Destr. Insp. 2013, 62, 527-528. (In Japanese) 